\title{
Análisis de las Teorías Organizacionales por la metáfora del organismo y la Evolución de Lamarck
}

\author{
Brenda Yuriria Bejarano Lugo ${ }^{1}$
}

\section{RESUMEN}

El propósito del presente documento es el análisis de las teorías organizacionales respecto a la evolución de la generación de conocimiento a la gestión organizacional. Dicho análisis comprende desde la década de 1970 a 1990, particularmente teorías que se derivan del enfoque de sistemas abiertos promovido por la Teoría General de Sistemas de Betarlanffy (1986). Por tal motivo el método de análisis es a través de la reflexión metafórica de organismo sugerida por Morgan (1990). De igual manera se considera que la comparación de dicha evolución debe de ser en el mismo sentido (e.i como un organismo), por lo que el punto de referencia es la primera teoría biológica de la evolución transformativa de Jean Baptiste Lamarck. Todo esto con la finalidad de simplificar la comparación evolutiva de las teorías, y en consecuencia proporcionar una nueva forma de entender las similitudes que guardan y la evolución.

Palabras Claves: Evolución, teoría, organizaciones

Recibido: 04 de noviembre de 2016

Aceptado: 10 de noviembre de 20016

1 Lic. En Administración de Empresas, Instituto Tecnológico de Sonora Cd. Obregón Sonora, México. Correo electrónico: yuririab@gmail.com 


\title{
Analysis of Organizational Theories by the metaphor of the body and the Evolution of Lamarck
}

\begin{abstract}
The purpose of this paper is the analysis of organizational theories about the evolution of the generation of knowledge to organizational management. This analysis ranges from 1970 to 1990, particularly theories derived from open systems approach promoted by the General Systems Theory Betarlanffy (1986). Therefore the analysis method is through the metaphoric reflection body suggested by Morgan (1990). Similarly, it is considered that the comparison of such developments must be in the same direction, so the benchmark is the first biological theory of transformational evolution of Jean-Baptiste Lamarck. This to simplify comparison of evolutionary theories, and thus provide a new way to understand the similarities that keep and developments that have taken for greater understanding and conceptualization.
\end{abstract}

Keywords: Evolution, theory, organizations. 


\section{INTRODUCCIÓN}

Las organizaciones en el mundo están presentes por doquier, el desarrollo de la humanidad y las múltiples actividades que van surgiendo como producto de sus necesidades básicas y superfluas han aumentado el número de las organizaciones y su complejidad hasta fechas actuales. El funcionamiento óptimo de las mismas impacta cada aspecto importante de las sociedades, siendo estos económicos, políticos, sociales, tecnológicos y ambientales, a nivel local, nacional y global, por tanto la magnitud e impacto de estas las hace sumamente importantes, motivo por el cual el estudio de ellas es básicamente trascendental.

Las organizaciones sean, organismos no lucrativos, instituciones públicas y empresas privadas son diferentes en gran parte de sus estructuras procesos fundamentos y elementos que las conforman, pero también guardan grandes similitudes, la comprensión de estas entidades en su totalidad, así como la evolución de las teorías que abordan o tratan de escrutarlas para lograr su entendimiento, es pieza medular en la gestión de las mismas, por tanto el siguiente documento tiene como objetivo ofrecer una perspectiva de la evolución de las teorías y modelos organizacionales, básicamente del periodo de la década 1970 a la década de 1990, es decir desde el cambio de paradigma del sistema cerrado al sistema abierto, con la Teoría General de Sistemas (TGS) sugerida por Betarlanffy (1986). Para el efecto de dicho análisis, se tomarán a algunas teorías así como algunos representantes los cuales se pueden observar en la Tabla 1.

El análisis propuesto está basado en la evolución de estas teorías a partir del conocimiento generado para entender a las organizaciones enfocado a cierto aspecto, por tanto el método de análisis es a través de una reflexión metafórica dada por Morgan (1990) el cual sugiere tres metáforas que rigen a las teorías. Para este autor existe una tricotomía que comprende, la mecánica, orgánica y la cultural. Siendo la Orgánica, la cual este autor designa a la teoría de sistemas, sin embargo en el presente ensayo se generalizará esta metáfora en todas las teorías y modelos expuestos, por pertenecer al enfoque sistémico, para poder así compararlas de manera general.

Debido a lo anterior el presente documento abordará la metáfora de la organización como organismo vivo ya que como se había previamente comentado a partir de la metateoría de sistemas (Betarlanffy, 1986) se considera el cambio de paradigma, donde la organización como sistema abierto permite nuevos enfoques, administrativos -estructurales y funcionales de las teorías organizacionales-, así pues al considerar a la organización como un ser vivo, por ende la comparación de dicha evolución debe de ser en el mismo sentido, por tal motivo se tomará como punto de referencia la primera teoría biológica de la evolución transformativa de Jean Baptiste Lamarck, que según algunos autores es el verdadero fundador de la teoría de la descendencia, siendo una equivocación atribuir a Darwin dicho Origen (e.g. Ernst Haeckel,1897). Para Lamarck una de las ideas claves de la evolución, es la existencia de una fuerza que dirige inconsciente o conscientemente a los seres vivos a volverse más complejos, el impulso de mejorar, todo esto se da por que el medio ambiente donde se desarrollan los organismos está en continua transformación, por tanto estas últimas también se transforman en función de esa adaptación tal como se puede observar en el siguiente párrafo:

Lamarck (1809) estudiando a los animales de todas las clases se encuentra otras cosas que examinar que la que se refiere a su composición. El producto de las circunstancias como causas que ocasionan nuevas necesidades, el de las necesidades que crea los hábitos y las inclinaciones, los resultados del empleo aumentado o disminuido de tal o cual órgano, los medios de que la naturaleza se sirve para conservar y perfeccionar todo lo que ha sido adquirido en la organización, etc. (p. XVIII). 
Además de esta consideración, el autor afirma que la evolución es un proceso de cambio continuo $\mathrm{y}$ progresivo que es transmitido de un organismo a otro. Ahora bien ya aclarado ese punto el cual es pieza clave en el presente análisis, se aborda entonces la conceptualización de especie, puesto que si se abordará la evolución tal como la planteó este biólogo, se debe de comparar entre especies, refiriéndose a esta como los seres que guardan similitudes y se reproducen entre ellos; esta acepción es retomada, en el sentido de que se utilizará como especies a las teorías que forman parte de algún aspecto similar en las teorías organizacionales, pero al mismo tiempo retomando las palabras claves en la teoría evolucionista de Lamarck, las cuales son necesidad-función y órgano. Es importante hacer énfasis que el órgano, bajo el esquema que se pretende analizar de las teorías y modelos organizacionales desde tres componentes que se consideran importantes para la conceptualización siendo estos: personas, procesos y herramientas. Por tal motivo para determinar si existe evolución se agruparan las teorías que se consideren pueden ser comparadas por pertenecer a la misma especie, y se dividirán en estos tres preceptos.

\section{La clasificación de las teorías según su especie}

Comoyaseplanteó en el párrafo anterior, la comparación entre las teorías retomadas en este documento pretende simplificar sus aspectos más notorios para de esa forma poder hacer una comparación evolutiva, por lo tanto es necesaria agruparlas. Por lo tanto retomando la conceptualización de Lamarck de especie, básicamente como organismos que tienen similitudes es que se hizo la siguiente agrupación que comprende de 4 especies y de denominaron por considerar los aspectos más importantes para le generación de esa teoría así como las similitudes de estos entre ellas, quedando de la forma que se describe a continuación.

Interactivas y adaptativas tomándose en cuenta como necesidad principal sobrevivir y adaptarse buscando la legitimidad, y aunque pudiera ser el objetivo esencial de todas las teorías aquí presentes, puesto que derivan del enfoque sistémico se considera estas están un grado más enfocadas en este aspecto que el resto. El segundo grupos es dinámicas en su proceso de gestión, puesto que básicamente ese es el aspecto general que sostiene estas teorías como forma de fomentar ese dinamismo en la gestión y de ese modo lograr cambios en los procesos, normas promoviendo la descentralización del poder, la disminución de la burocracia y las interacciones entre individuos, situaciones y procesos a favor de la organización. El tercer grupos también promueve el dinamismo, pero esto en favor de la generación de conocimiento e innovación en procesos productos funciones, etc.; de la organización. El cuarto y último grupo se denominó las competitivas, puesto que el objeto principal es la obtención de la competitividad y una posición privilegiada en los mercados, como líderes, y en esa búsqueda las prácticas de la gestión se enfocan en la ventaja competitiva así como el análisis de sus recursos tanto externos como internos.

De igual manera, para efectos de comparación entre las teorías, y bajo el esquema de la evolución de los organismos vivos sugerido por Lamarck (1809) se procuró conceptualizar en pocas palabras la necesidad del grupo o de la teoría, según fuera el caso, pues en algunas había incluso dicotomías un ejemplo de ello la teoría de recursos y capacidades, enfocada a el ambiente interno en su gran parte, y las herramientas sugeridas por Porter basadas en el ambiente externo; Sin embargo al visualizar de forma completa la teoría estos extremos sugieren complementarse. En cuanto a la función principal se describió en base a esa necesidad, y el órgano, como en las teorías organizacionales y modelos aquí presentados se puede variar principalmente en tres aspectos sumamente importantes: Personas, procesos y herramientas, estos fueron, los tres principales componentes que se establecieron como los más importantes para llevar a cabo las funciones de las teorías. Para facilitar la comprensión se puede ver en la siguiente tabla. 
Tabla 1. Clasificación de las teorías bajo el esquema de organismos biológicos

\begin{tabular}{|c|c|c|c|}
\hline Especie & $\begin{array}{l}\text { Necesidad que busca cubrir } \\
\text { la teoría }\end{array}$ & $\begin{array}{c}\text { Función principal en base } \\
\text { a la necesidad }\end{array}$ & $\begin{array}{c}\text { Órgano (personas, proceso } \\
\text { herramientas) }\end{array}$ \\
\hline $\begin{array}{l}\text { Interactivas y adaptativas } \\
\text { Teoría neoinstitucional (Powell } \\
\text { y Dimaggio, 1983) }\end{array}$ & $\begin{array}{l}\text { Interacción y aceptación } \\
\text { del entorno social y de la } \\
\text { competencia. Disminución de } \\
\text { la burocracia }\end{array}$ & $\begin{array}{l}\text { daptarse al entorno y } \\
\text { obtención de legitimidad. }\end{array}$ & $\begin{array}{l}\text { Proceso de cambio hacia la } \\
\text { aceptación }\end{array}$ \\
\hline $\begin{array}{l}\text { Relaciones } \\
\text { interorganizacionales. } \\
\text { (Evan, 1967) }\end{array}$ & & $\begin{array}{l}\text { Conocer las relaciones e } \\
\text { interacciones importantes } \\
\text { para la organización }\end{array}$ & $\begin{array}{l}\text { Herramienta de } \\
\text { Análisis interorganizacional }\end{array}$ \\
\hline $\begin{array}{l}\text { Dinámicas en sus procesos de } \\
\text { gestión } \\
\text { Anarquías organizadas. } \\
\text { Cohen, March, y Olsen, 1972) }\end{array}$ & $\begin{array}{l}\text { Flexibilidad en los procesos } \\
\text { organizacionales, disminución } \\
\text { de la burocracia }\end{array}$ & $\begin{array}{l}\text { Dinamismo organizacional } \\
\text { en función del cambio para } \\
\text { mejorar }\end{array}$ & Procesos organizacionales flexibles \\
\hline $\begin{array}{l}\text { Teoría del caos (Thietart, y } \\
\text { Forgues, } 1995 \text { y McBride, } \\
\text { 2005) }\end{array}$ & $\begin{array}{l}\text { Flexibilidad en los procesos } \\
\text { organizacionales, disminución } \\
\text { de la burocracia }\end{array}$ & $\begin{array}{l}\text { Dinamismo organizacional } \\
\text { en función del cambio para } \\
\text { mejorar }\end{array}$ & Procesos organizacionales flexibles \\
\hline $\begin{array}{l}\text { Dinámicas en la creación del } \\
\text { conocimiento e Innovadoras } \\
\text { Gestión del conocimiento } \\
\text { Nonaka y Takeuchi, (1995) }\end{array}$ & $\begin{array}{l}\text { Innovación y creación de } \\
\text { conocimiento }\end{array}$ & $\begin{array}{l}\text { Gestionar el conocimiento } \\
\text { tácito y explicito }\end{array}$ & $\begin{array}{l}\text { Las personas como la fuente } \\
\text { de creación de conocimiento e } \\
\text { innovación. }\end{array}$ \\
\hline $\begin{array}{l}\text { Desarrollo Organizacional } \\
\text { Richard Beckhard, 1963) del } \\
\text { cambio en la organización }\end{array}$ & $\begin{array}{l}\text { El cambio organizacional de } \\
\text { forma eficiente }\end{array}$ & $\begin{array}{l}\text { Cambiar la organización } \\
\text { para su desarrollo }\end{array}$ & $\begin{array}{l}\text { Las personas como agentes } \\
\text { principales }\end{array}$ \\
\hline $\begin{array}{l}\text { Competitivas Administración } \\
\text { estratégica (Mintzberg,, 1978) }\end{array}$ & $\begin{array}{l}\text { Formular estrategias } \\
\text { Diferenciación y ventaja } \\
\text { sobre los competidores }\end{array}$ & $\begin{array}{l}\text { Formulación de la } \\
\text { estrategia y creación de la } \\
\text { ventaja competitiva }\end{array}$ & $\begin{array}{l}\text { Proceso de formulación de } \\
\text { estrategias. }\end{array}$ \\
\hline $\begin{array}{l}\text { Teoría de Recursos y } \\
\text { capacidades (Barney, 1991) }\end{array}$ & & $\begin{array}{l}\text { Identificar los recursos } \\
\text { potenciales generadores de } \\
\text { la ventaja competitiva, así } \\
\text { como la ventaja competiti- } \\
\text { va sostenible }\end{array}$ & $\begin{array}{l}\text { Herramienta de análisis interno } \\
\text { para identificar los atributos y los } \\
\text { recursos potenciales para la formu- } \\
\text { lación de estrategias }\end{array}$ \\
\hline $\begin{array}{l}\text { Ventaja competitiva (Porter, } \\
\text { 1979) }\end{array}$ & & $\begin{array}{l}\text { Formular estrategias que } \\
\text { lleven a la ventaja compe- } \\
\text { titiva }\end{array}$ & $\begin{array}{l}\text { Herramienta de análisis interno y } \\
\text { externo para identificar los atribu- } \\
\text { tos y los recursos potenciales }\end{array}$ \\
\hline
\end{tabular}

Fuente. Elaboración propia

Análisis de la Evolución de las teorías

\section{Teoría neo institucional y Relaciones interorganizacionales}

Cabe mencionar que es complejo simplificar las teorías y los modelos los cuales atañen muchos aspectos organizacionales, sin embargo la intención del documento es hacer énfasis en lo que se considera más trascendental para el análisis de la evolución según cada teoría o modelo expuesto en el presente documento. Para iniciar con el análisis como se puede observar la teoría neoinstitucional propuesta por Powell y Dimaggio (1983), como se había comentado previamente se agrupó con relaciones interorganizacionales como parte de una misma especie, nombrándolas por su similitud y características principales en función de la teoría de la organización como, interactivas y adaptativas tomándose en cuenta como necesidad principal sobrevivir y adaptarse buscando la legitimidad. 
Para DiMaggio y Powell (1983) El proceso es una "estructuración" y consta de cuatro partes (1) el crecimiento de la interacción entre las organizaciones en el campo, (2) emergencia entre sus patrones de coalición y estructuras de dominación, (3) un incremento en la carga de información en las cuales las organizaciones tienen que enfrentarse y por último (4) el desarrollo del conocimiento recíproco entre los participantes de un conglomerado de organizaciones que están relacionados en una empresa en común. Existen ciertos aspectos básicamente estructurales que los actores perciben como racionales, ciertas características que se pueden modelar, copiar imitar o por mecanismos normativos o coercitivos tienen a generar el cambio hacia la institucionalización existen dos tipos el competitivo: que implica presiones dirigidas a los resultados similares a la competencia de mercado, y el isomorfismo institucional que es el que más se enfocó.

De este último hizo siguiente clasificación de tres tipos, el primero era el coercitivo se refería básicamente influencia de la política, repuestas a los mandatos gubernamentales un ejemplo serían los fabricantes que optan por usar tecnologías para cumplir con las regulaciones ambientales, el segundo que es el mimético el cual parte de la incertidumbre, ante la ambigüedad del medio ambiente y los propios objetivos del tipo de organización tienden a modelar otras organizaciones puesto que la percibe como segura y legitima y por último el normativo donde las redes o normas influyen en el cambio; así pues las organizaciones adoptan practicas modelos y hasta tecnologías de otras organizaciones y es ahí donde inicia ese cambio o isomorfismo. Cabe mencionar que en cuestiones de tecnología las organizaciones que imitan se ahorran el costeo de pruebas que pudieron haber tenido las organizaciones de quienes se copiaron. Al respecto Scott (1987) hace hincapié que esta teoría está enfocada básicamente en tres cuestionamientos, ¿Cómo son?, ¿Cómo actúan? Y ¿Cómo cambian? Pero no como surgen ni lo que pudiera desencadenar los procesos de institucionalización. Para lo cual lo expuesto por Evan en el mismo año pudiera ser un punto de partida para mejorar la perspectiva de la institucionalización.

Evan (1967) tomó la propuesta, de órbita del Rol desarrollado por Merton (en Evan; 1967), el cual se refiere a las interrelaciones, que desempeña un individuo en función de un status, un ejemplo de ello pueden ser los gerentes de ventas de una organización buscando vender a otra empresa, los cuales deben establecer contacto con el personal de compras o los encargados de tomar la decisión de comprar. Donde la dificultad radica precisamente en el seguimiento de lo contractual, pues las otras organizaciones también cuentan con normas, que en ocasiones no convergen con las normas de la empresa del departamento de ventas. Así pues Evan (1967) propone la órbita pero de la organización donde el status no es en sí de un puesto en particular sino de la organización con respecto a otras organizaciones, en donde la organización específicamente analizada es denominada como organización focal.

Evan (1983) Comenta que el objetivo principal de su perspectiva es proporcionar un marco de referencia que aportaría una explicación que ayudaría a entender:

(1) · la estructura interna de la organización focal; (2) su grado de autonomía en la toma de decisiones; (3) su grado de eficacia o del alcance de las metas; (4) su identidad, es decir su autoimagen y su imagen pública; (5) el flujo de información que va de la organización focal a los elementos de su configuración organizacional y inversamente; (6) el flujo de personal que va de la organización focal a los elementos de su órbita organizacional y recíprocamente y (7) las fuerzas que impulsan a la organización focal a cooperar o competir con elementos de su órbita, a coordinar o fusionar sus actividades con otras organizaciones o disolverse. (p.201).

Podría considerarse que en la comparación de estas dos teorías existe una evolución puesto que hay un nuevo conocimiento adquirido necesario por parte de esta 
perspectiva en función de un análisis que podría ayudar a complementar lo que la teoría interorganizacional sugería con las relaciones e interacciones con otras organizaciones, así como de sus implicados, donde la teoría institucional expone mediante su interacción con otras organizaciones tratando de buscar una legitimidad, la aceptación social, así como una adaptación a las pautas, los ambientes turbulentos y cambiantes así como a la normatividad impuestas entre organizaciones.

\section{Teoría del Caos, Anarquías Organizadas.}

La especie a la que pertenece este grupo es dinámicas en su proceso de gestión. Iniciando con la conceptualización de la teoría del caos, Según Kellert (1993; en McBride, 2005) dice que se puede definir como el estudio del comportamiento inestable de los sistemas dinámicos no lineales, es decir no es notoriamente pronosticable o previsible. Si se aplica a la gestión de las organizaciones Dolán García, y Auerbach (2003; en Pidal, 2009) comentan que la teoría del caos intenta comprender la relación entre el orden y el desorden, de este modo es viable del orden llegar al caos y viceversa.

La Teoría del caos según Thierart y Forgues (1995) surge como una nueva perspectiva o enfoque del antiguo paradigma el cual se asemejaba a una serie de recetas a seguir paso paso, considerando a la organización como un ente estático, el cual era un problema para los gerentes pues aunque la teoría se concebía como algo fácil, en la práctica esto era algo bastante difícil. Por lo tanto dicha teoría hace énfasis en lo importante que puede ser el dinamismo para las organizaciones, el cual les podría ayudar a innovar crecer y desarrollarse. En esta misma línea anarquías organizadas un modelo teórico el cual hace a una metáfora de botes de basura sugerido por Cohen, March, y Olsen, (1972) donde también abordan la ambigüedad así como la dificultad del control en las organizaciones sobre todo al momento de tomar decisiones y la ejecución de las mismas, donde la burocracia limita pues la gestión que sugiere el modelo es descentralizado, menos jerarquizado; por tanto entre estas dos teorías también se considera que hay una evolución, desde la consideración de la ambigüedad y la dificultad en la gestión de algunas organizaciones, mismo que fue desarrollado en la teoría de caos, como un resultado de la teorización hecha en anarquías organizadas, ambas perspectivas sugieren que las organizaciones no son estáticas ni rígidas, pero que en su continuo cambio y la ambigüedad que se suscitan de las interacciones de sus participantes así como las circunstancias y situaciones pueden servir para conservar el principio de neguentropia característica de los sistemas abiertos que se refiere a que el dinamismo de estos los hace seguir vivos. Existe evolución al sugerir procesos organizacionales flexibles menos burocratizados, en el caso de anarquías organizadas en las elecciones de decisiones, sin embargo era conceptualizada en universidades, la teoría del caos amplio el enfoque hablando de todo tipo de organizaciones, y no solo el proceso de decisiones si no en si en todo los procesos que se involucran en las organizaciones.

\section{Teoría de Gestión del conocimiento y Desarrollo organizacional.}

Este tercer grupo de teorías tiene como objetivo principal, la generación de innovación en procesos, productos y funciones de las organizaciones, donde la innovación es atributo que se valora, pero para poder obtenerlo se debe de obtener un cambio y/o gestionar el conocimiento. Nonaka y Takeuchi (1995), desarrollaron un modelo para gestionar el conocimiento, donde la relataron la importancia del conocimiento explicito (estructurado, más fácil de ordenar transferir ya transformar) y el tácito(el cual es más difícil de externalizar pues los individuos lo poseen, estos pueden ser experiencias, creencias valores formación etc) y como se dan a través de una interacción social (explicada en su espiral del conocimiento) donde lo más recomendable es que se use como generación de 
conocimiento a favor de la organización; Por su parte Beckhard (1969): estableció el concepto de DO donde para dar el cambio en las organizaciones es necesario hacerlo a través de un proceso holístico que incluya tanto a administradores como al personal de mandos medios y operativo (todos los niveles organizacionales) en donde el comportamiento de los individuos es clave para la consideración del desarrollo y alcanzar la eficiencia de la organización, en el proceso del cambio se deben de tomar en cuenta factores como la cultura, liderazgo por mencionar algunos. La evolución aquí radica en el hecho de considerar a las personas como fuente de innovación y cambio, en el Desarrollo organizacional ya hacían notoria esta contribución sin embargo, en la gestión del conocimiento la perspectiva se amplió y la fundamentación dada por Nonaka y Takeuchi (1995), ha sido enriquecedora para la gestión organizacional incluso en las dicotomías expuestas en su obra, la espiral del conocimiento, donde a través de la socialización se puede externar e interiorizar el conocimiento tácito y explícito y la sugerencia del hipertexto middle up, como modelo de la gestión favorecedor de la creación de conocimiento, por mencionar algunas de las contribuciones de estos exponentes.

\section{Administración estratégica, Teoría de Recursos y Capacidades, Ventaja competitiva.}

Este último grupo de análisis fue nombrado competitivo, por que el enfoque va directamente a generar competitividad, la creación de las estrategias en función de las ventajas competitivas. Aunque existen múltiples teorías y modelos que se derivan de administración estratégica, para fines del presente documento se toma particularmente como fuente de análisis en primer lugar la aportación trascendental de Mintzberg (1978), por considerarse como uno de los precursores de este enfoque dejando atrás la planeación estratégica que daba una perspectiva estática y burocratizada de la formulación de la estrategia, por, una nueva perspectiva más flexible y ad hoc a los contextos cambiantes y turbulentos Mintzberg (1978) criticó la postura de Chandler (1962), al decir que la estrategia no necesariamente se ligaba a la estructura y más que formulada era formada, esta nueva perspectiva sugería una nueva forma de pensar, crear, usar y estudiar la estrategia, esta corriente filosófica sigue aún en construcción, sin embargo se han hecho grandes contribuciones teóricas, ejemplo de ellas es la teoría de recursos y capacidades de Barney (1991) quien desarrolló en esta teoría las condiciones o atributos por las que los recursos de la empresa pudieran transformarse en la ventaja competitiva sostenible, desde el punto de vista interno, fortalezas y debilidades, gestionando así de forma razonable sus recursos y capacidades, se enfocó precisamente a esto puesto que consideraba que la literatura que se estaba suscitando en ese momento estaba más encaminada a valuar los aspectos externos que tenían que ver directamente con el medio ambiente. Con lo anterior el autor se refería a Porter (1979) quien en definitiva hizo aportaciones basadas en el contexto externo básicamente de la industria, y la generación de las ventajas competitivas, el analizó la industria y lo expuso por medio de su conceptualización de las cinco fuerzas, donde se diagnostican y definen la rentabilidad de una industria y de igual forma también son importantes para la formulación de la(s) estrategia(s); Así pues en base a este tercer grupo existe una evolución notoria desde la conceptualización de Mintzberg(1978), sin embargo como se puede notar los autores hablan de aspectos distintos, pero que cada uno de ellos es importante para la formación (Minztberg, 1978) o formulación de la estrategia (Porter, 1979) aun así se considera evolución puesto que se ha ampliado o modificado el órgano, en este caso personas, procesos y herramientas en función de la perspectiva que sugiere la Administración estratégica, detección de oportunidades explotación de los recursos, dirección y ejecución de la estrategia, sin dejar de lado algunos procesos que si deben estar sistematizados como lo sugiere la planeación estratégica. 


\section{CONCLUSIÓN}

Se ha tratado de analizar las teorías expuestas bajo el enfoque de sistemas como organismos biológicos, para considerar su evolución, los aspectos trascendentales de las teoría de sistemas, y todo lo anterior de tal manera que se buscó simplificar el análisis por medio de la metáfora y bajo el esquema evolutivo de los organismos expuesto por Lamarck (1809). cabe mencionar que la comparación está hecha desde una teoría a otra y en base a la agrupación por las consideraciones asignadas, sin embargo la influencia que las teorías organizacionales han tenido unas con otras es algo que no se puede ignorar, es decir hay una multiplicidad de influencias entre estas y todas las teorías organizacionales existentes ello se sabe por obviedad, sin embargo el objetivo del documento era hacer una reflexión simple de algo tan complejo como son estas teorías, no se subestiman en ningún momento otras aportaciones y contribuciones de las mismas, puesto que la conceptualización de ellas abordan un gran número de aspectos que competen a las organizaciones y su funcionamiento, mismos que sería sumamente complejo para acotarlos en este ensayo. Por otro lado la reflexión de la evolución de las mismas era una tarea difícil puesto que a simple vista parecen ser totalmente diferentes pues abordan casi de manera unilateral aspectos organizacionales, por tal motivo se procuró simplificarlas en las similitudes que guardan; y bajo esta perspectiva se denota que efectivamente existe evolución, puesto que hay una transformación y un cambio que responde a los contextos en los cuales se fueron desarrollando las teorías y modelos propuestos, así como retomando puntos de las teorías antecesoras y buscando agregar lo que había faltado, sin embargo como bien se sabe, la organizaciones son complejas tienen multiples interacciones, son ambiguas, y encontrar las multiples conceptualizaciones de cada uno de sus aspectos, es una tarea bastante difícil, la cual solo podrá ser descrita, si como hasta el momento las teorías buscan la integración y entendimiento de cada uno de sus aspectos, quizás algún día se pueda integrar de manera holística en fragmentos cada una de las aportaciones hechas por las teorías a este gran sistema complejo que es la organización, sin embargo hasta el momento queda seguir buscando desde cada una de sus perspectivas para poder ampliar la visión del gran sistema que representa.

\section{REFERENCIAS}

Barney, J. B. (1991). Firm Resources and Sustained Competitive Advantage. Journal of Management, 17 (1), 99-120.

Beckhard, R. (1969) Organization Development: Strategies and Models, Addison-Wesley, Reading, MA.

Bertalanffy, L. V. (1986). Teoría General de Sistemas. México: Fondo de Cultura Económica.

Chandler, A. D. (1962). Strategy and structure: Chapters in the history of the American enterprise. Massachusetts Institute of Technology Cambridge

Cohen, M. , March, J., \& Olsen, J. (1972). A garbage can model of organizational choice. Administrative science quarterly, 1-25.

Cohen, M., March, J., \& Olsen, J. (2011). El bote de basura como modelo de elección organizacional. Gestión y política pública, 20(2), 247-290.

DiMaggio, P., \& Powell, W. (1983) "The iron cage revisited: Institutional Isomorphism and collective rationality in organizational fields" American Sociological Review, 48:147-160.

Drucker, P. F. (1955). "Management Science" and the Manager. Management Science, 1(2), 115-126.

Drucker, P. F. (1955b). The management horizon. The Journal of Business, 28(3), 155-164.

Drucker, P. F. (1958). Business objectives and survival needs: notes on a discipline of business enterprise. The Journal of Business, 31(2), 81-90.

Evan, W. M. (1967). La órbita de la organización: Hacia una teoría de las relaciones interorganizacionales. Buenos Aires: OMEB

Haeckel, E. H. P. A. (1897). The evolution of man (Vol. 1). Appleton. Journal of Sociology, vol. 83: 340- 
363.1977.

Kast, F. E., \& Rosenzweig, J. E. (1988). Administración en las organizaciones, enfoque de sistemas y de contingencias (4ta. Ed.). México: McGraw Hill.

Katz, D., \& Kahn, R.L. (1977). Psicología Social de las Organizaciones. México: Trillas.

Lamarck, J. B. (1809). Filosofía zoológica. Presentación de Adriá Casinos. Editorial Alta Fulla. Mundo Científico. Barcelona.

McBride, N. (2005). Chaos theory as a model for interpreting information systems in organizations. Info Systems J, 15 (1), 233-254.

Meyer \& Rowan. Institutionalized organizations: formal structure as myth and cerernony. American

Mintzberg, H. (1978). Patterns in strategy formation. Management science, 24(9), 934-948.

Morgan, G. (1990). Imágenes de la organización. Madrid: RA-MA Editorial. La organización como máquina. En Morgan(1990). Imágenes de la administración, 9-29
Nonaka, I., \& Takeuchi, H. (1995). The knowledgecreating company: How Japanese companies create the dynamics of innovation. Oxford university press.

Pidal, M. (2009). La Teoría del Caos en las Organizaciones. cuadernos unimetanos, 29-33.

Porter, M. How competitive forces shape strategy. Harvard Business Review, March-April 1979, 5 7, 137-156.

Porter, M., Campos, E., Moreno, C., \& Sánchez, M. (2010). Ventaja competitiva: creación y sostenibilidad de un rendimiento superior. Editorial Continental, México.

Rumelt, R., Schendel, D., \& Teece, D. J. (1991). Strategic management and economics. Strategic management journal, 12(S2), 5-29.

Scott, W. R. (1987). The adolescence of institutional theory. Administrative science quarterly, 493-511. Thietart, R, \& Forgues, B. (1995). Chaos Theory and Organization. Organization Science, 6 (1), 19-31. 